\title{
On the twin paradox in static spacetimes: I. Schwarzschild metric
}

\author{
Leszek M. Sokołowski
}

Received: 8 October 2011 / Accepted: 6 February 2012 / Published online: 18 February 2012

(C) The Author(s) 2012. This article is published with open access at Springerlink.com

\begin{abstract}
Motivated by a conjecture put forward by Abramowicz and Bajtlik we reconsider the twin paradox in static spacetimes. According to a well known theorem in Lorentzian geometry the longest timelike worldline between two given points is the unique geodesic line without points conjugate to the initial point on the segment joining the two points. We calculate the proper times for static twins, for twins moving on a circular orbit (if it is a geodesic) around a centre of symmetry and for twins travelling on outgoing and ingoing radial timelike geodesics. We show that the twins on the radial geodesic worldlines are always the oldest ones and we explicitly find the the conjugate points (if they exist) outside the relevant segments. As it is of its own mathematical interest, we find general Jacobi vector fields on the geodesic lines under consideration. In the first part of the work we investigate Schwarzschild geometry.
\end{abstract}

Keywords Twin paradox $\cdot$ Static spacetimes $\cdot$ Jacobi fields $\cdot$ Conjugate points

\section{Introduction}

The twin paradox is well understood in Minkowski spacetime whereas in curved spacetimes less is known about generic features of the twins following various worldlines and recent works studying various special cases are quite numerous (see e.g. [1-6] and references therein). Abramowicz, Bajtlik and Kluźniak investigated a couple of static spacetimes where the absolute rest is defined by the unique timelike Killing

\footnotetext{
L. M. Sokołowski ( $)$

Astronomical Observatory, Jagiellonian University, Orla 171, 30-244 Kraków, Poland

e-mail: lech.sokolowski@uj.edu.pl

L. M. Sokołowski

Copernicus Center for Interdisciplinary Studies, Kraków, Poland
} 
vector field and a stationary axially symmetric spacetime where the standard of rest is determined by the rest frame of the zero angular momentum observers (ZAMO) and calculated the proper times for the twins orbiting on the same circular orbit with different constant linear velocities. From the case of these circular and stationary motions they have drawn a conclusion which being generalized to other worldlines is actually a conjecture: 'in all situations in which the absolute motion may be defined in terms of some invariant global properties of the spacetime, the twin who moves faster with respect to the global standard of rest is younger at the reunion, irrespectively to twins' accelerations' $[7,8]$. It is the purpose of the present work to show that in general the conjecture is false.

We first make a comment on the concept of rest. Geometrically an absolute standard of rest is provided by the timelike Killing vector field (if it exists and is unique), one may, however, consider a universal standard of rest based on the synchronous (i.e. comoving, i.e. normal Gauss) frame of reference which is freely falling in the gravitational field. The comoving frame may cover only a part of the spacetime (as it develops, besides some special cases, coordinate singularities) and the distances between freely falling bodies change in time, nevertheless to some extent the bodies may be treated as being at rest. Accordingly, if radially freely falling bodies in Schwarzschild spacetime are supposed to be at rest, then a static Killing observer and an observer on a circular geodesic (i.e. an observer orbiting around the central body) are moving with respect to them and, as is shown below, their proper times are shorter than those at rest. This case supports the Abramowicz's, Bajtlik and Kluźniak (ABK) conjecture. However, this notion of rest is not unambiguous. In fact, the observers on circular orbits are freely falling too and if they are regarded as staying at rest then the radially falling observers and the static Killing ones are in respective motion and their proper times are larger than those of the motionless (i.e. orbiting on circles) observers. We therefore assume that the state of rest is determined by the unique Killing field.

The problem of which twin travelling between two given spacetime points is older is of purely geometrical nature and the answer to the question of which worldline makes a twin the oldest one (assuming a large number of twins following various worldlines with the same endpoints) is implicitly well known as it may be found in advanced monographs. Imprecise answer is even contained in an intermediate level textbook [9]: 'for a really reliable answer one has to know how to deal with accelerated systems; here general relativity is to be asked, and answer is: yes, travelling (deviating from geodesic motion) keeps you younger'.

A rigorous formulation and solution of the problem is following. Let $U$ be a convex normal neighbourhood of a point $p$ in any Lorentzian spacetime. Then the following proposition holds:

Proposition 1 (Proposition 4.5.3 in [10]) In any convex normal neighbourhood $U$, if $p$ and $q$ can be joined by a timelike curve then the unique timelike geodesic connecting them has length strictly greater than that of any other piecewise smooth timelike curve between the points.

Schwarzschild spacetime is misleading in this context. Consider two twins: A is at rest at a point $r=r_{0}>3 M$ (in the standard Schwarzschild coordinates) and B moves on a timelike geodesic line with a circular orbit in the space at $r=r_{0}$. Initially the 
twins are at a spacetime point $P_{0}\left(t=t_{0}, r=r_{0}\right)$, then B flies away, makes a full circle around the centre and meets A again at $P_{1}\left(t=t_{1}, r=r_{0}\right)$. A simple calculation shows that the proper time $s_{A}$ (i.e. the worldline length) of A between $P_{0}$ and $P_{1}$ is longer than the geodesic proper time $s_{B}$ of B,

$$
\frac{s_{A}}{s_{B}}=\frac{1}{\sqrt{1-\left(\frac{v}{c}\right)^{2}}}=\left(\frac{r_{0}-2 M}{r_{0}-3 M}\right)^{1 / 2}>1,
$$

where $v$ is B's velocity with respect to the static A [7,8]. This case gave rise to the conclusion that the twin who moves faster (and without acceleration) is younger than the static twin (subject to acceleration). However this simple example merely shows that $P_{1}$ does not belong to a convex normal neighbourhood of $P_{0}$. In fact, there is at least one timelike geodesic line joining $P_{0}$ and $P_{1}$ whose length is larger than that of B.

Here the key notion is that of conjugate points and for the reader's convenience we briefly recall the relevant facts. Let $\gamma$ be a timelike geodesic with tangent unit $u^{\alpha}$. Any vector field $Z^{\mu}(s)$ which is a solution to the geodesic deviation equation on $\gamma$ is called a Jacobi field on $\gamma$. A pair of points $p$ and $q$ on $\gamma$ are said to be conjugate if there exists a Jacobi field $Z^{\mu}$ on $\gamma$ which is not identically zero and $Z^{\mu}(p)=Z^{\mu}(q)=0$ ([10] chapter 4). Points $p$ and $q$ are conjugate if infinitesimally nearby geodesic intersects $\gamma$ at both $p$ and $q$. Existence of conjugate points on a geodesic shows that it is neither the unique geodesic nor the longest curve connecting its endpoints. More precisely, if a timelike geodesic $\gamma$ joining points $p_{1}$ and $p_{2}$ has a point $q$ conjugate to $p_{1}$ belonging to the segment $p_{1} p_{2}$, then there exists a nearby timelike curve $\tau$ (not necessarily a geodesic one) with endpoints $p_{1}$ and $p_{2}$, which is longer than $\gamma, s(\tau)>s(\gamma)$. Conversely, if there is no conjugate points on a timelike geodesic, then it is the longest curve between its endpoints, independently of whether the endpoints lie in some convex normal neighbourhood, or not. It is stated in:

Proposition 2 (Proposition 4.5 .8 in [10]) A timelike geodesic $\gamma$ has the maximal length from $p_{1}$ to $p_{2}$ if and only if there is no point conjugate to $p_{1}$ on the segment $p_{1} p_{2}$.

In some spacetimes, e.g. anti-de Sitter space, two given points may be connected by various timelike curves, but none of these attains maximal length. The longest curve always exists in globally hyperbolic spacetimes.

Proposition 3 (Proposition 9.4 .4 in [11]) Let $\left(M, g_{\alpha \beta}\right)$ be a globally hyperbolic spacetime. Let $p_{1}$ and $p_{2}$ be connected by timelike curves. Then there exists a timelike geodesic $\gamma$ from $p_{1}$ to $p_{2}$ having the maximal length.

The existence of conjugate points is determined by

Proposition 4 (Proposition 4.4.2 in [10]) If $R_{\alpha \beta} u^{\alpha} u^{\beta} \geq 0$ on a timelike geodesic $\gamma$ and if the tidal force $R_{\mu \alpha \nu \beta} u^{\alpha} u^{\beta} \neq 0$ at some point $p_{0}$ on $\gamma$, there will be conjugate points $p$ and $q$ on $\gamma$, providing that the geodesic can be extended sufficiently far. 
Returning to the problem of the rate of ageing of the twins one sees that neither the velocity (in stationary spacetimes) nor absence of acceleration (on geodesic worldlines) alone is sufficient to establish which twin will be older at the reunion. In a curved spacetime there is a great variety of motions and for a given pair of worldlines there is no generic criterion (if none of them is a geodesic free of conjugate points) stating which of these is longer; one must explicitly compute their lengths. Therefore besides studying case by case one can only determine the longest worldlines joining given two points in some spacetimes. This is the heart of the present work.

For a given pair of spacetime points one applies physical arguments to find out a timelike geodesic connecting them and seeks for conjugate points on it. In physically interesting spacetimes the strong energy condition holds implying $R_{\alpha \beta} u^{\alpha} u^{\beta} \geq 0$ and in most cases the tidal forces do not vanish, hence Proposition 4 indicates that the geodesic under consideration contains somewhere conjugate points. It is then crucial to establish whether the conjugate points belong to the relevant segment of the geodesic.

In this work we explicitly analytically compute location of conjugate points on physically distinguished timelike geodesic lines in a number of static spacetimes and for comparison in Robertson-Walker spatially flat universe. To establish that the given geodesic is not the maximal one it is sufficient to find out a particular Jacobi vector field on it which reveals conjugate points within the relevant segment. A more general problem is finding out a generic Jacobi field on the given geodesic curve and there is an opinion (private communication) of a few experts in the field that this work is worth doing it as it is of mathematical interest. We get general solutions of the geodesic deviation equation for worldlines followed by the twins considered in the problem of their ageing rates. In the first part of the work we discuss the most convenient form of the geodesic deviation equation for the Jacobi field and then deal with Schwarzschild spacetime.

\section{Timelike geodesics in static spacetimes and Jacobi fields}

We consider static spacetimes since there the orbits of the timelike Killing vector field are orthogonal to hypersurfaces of simultaneity and the notion of observers at rest is unambiguous. The case of standard of rest with respect to the ZAMO frames shows that stationary non-static spacetimes require a separate treatment [8]. In a static spacetime there always exists a coordinate chart in which the metric takes the form

$$
d s^{2}=g_{00}\left(x^{k}\right) d t^{2}+g_{i j}\left(x^{k}\right) d x^{i} d x^{j}
$$

and the timelike Killing field is $K^{\alpha}=\delta_{0}^{\alpha}, K^{\alpha} K_{\alpha}>0,{ }^{1} \alpha, \beta=0,1,2,3$ and $i, j$, $k=1,2,3$. An observer is at rest if his worldline coincides with one of the orbits of the Killing field, then his velocity is $u^{\alpha}=g_{00}^{-1 / 2} \delta_{0}^{\alpha}$. From the geodesic equation $(D / d s) u^{\alpha}=0$, where $D / d s$ is the absolute derivative along a curve with respect to its arc length, one immediately sees that a static observer worldline is a geodesic if and only if $g_{00}=1$. This is a very special case and none of the physically interesting

\footnotetext{
${ }^{1}$ For obvious reasons we choose the metric signature $(+---)$.
} 
static spacetimes satisfies this condition. We shall always denote by A the nongeodesic observer at rest.

We assume that the spatial coordinates $\left(x^{i}\right)$ are so chosen that $\left(\partial / \partial x^{1}\right) g_{00}>0$. The coordinate $x^{1}$ varies in the range $-\infty \leq a \leq x^{1} \leq b \leq+\infty$ and $x^{1}$ tending to $b$ corresponds to spatial infinity (if it exists). Due to these properties we dub $x^{1}$ into 'radial coordinate'. We then seek for two kinds of worldlines as possible geodesics. Firstly, 'circular orbits' with $x^{1}=$ const $\neq 0$ which are spatially closed and secondly 'radial worldlines' with $x^{2}$ and $x^{3}$ constant and $x^{1}$ varying in an subinterval $a_{0} \leq x^{1} \leq b_{0}$ of $(a, b)$. In the spacetimes studied in this work the radial curves are timelike geodesics.

In the physical interpretation we shall assume that the twin B follows a circular worldline and the twin $\mathrm{C}$ moves on a radial geodesic curve. ${ }^{2}$ The twin A stays at $x^{1}=a_{0}$ and in a coordinate time interval $\Delta t$ his proper time is $s_{A}=\sqrt{g_{00}} \Delta t$. The twin C's radial worldline emanates outwards from $x^{1}=a_{0}$, reaches a maximal height $x^{1}=b_{0}$, turns inwards and comes back to $x^{1}=a_{0}$ to meet again $\mathrm{A}$ and possibly $\mathrm{B}$. The corresponding proper time $s_{C}$ of the twin $\mathrm{C}$ may be greater than $s_{A}$; this is possible although the expression $g_{i j} d x^{i} d x^{j}<0$ for $d x^{i} \neq 0$ if $g_{00}$ grows sufficiently quickly in the interval $a_{0}<x^{1}<b_{0}$.

On a given radial or circular geodesic $\gamma$ the conjugate points can be determined directly from the definition: by finding a generic solution $Z^{\mu}(s)$ to the geodesic deviation equation

$$
\frac{D^{2}}{d s^{2}} Z^{\mu}=R_{\alpha \beta \gamma}^{\mu} u^{\alpha} u^{\beta} Z^{\gamma},
$$

where $u^{\alpha}=d x^{\alpha} / d s, u^{\alpha} u_{\alpha}=1$, is the tangent vector field to the geodesic. The Jacobi field is orthogonal to the velocity vector, $Z^{\alpha} u_{\alpha}=0$. Due to the presence of the absolute derivatives, Eq. (3) are very complicated ODEs of second order and to simplify them one applies the following approach ([10] chapter 4, [11] chapter 9). On $\gamma$ one introduces an orthonormal triad of spacelike vector fields $e_{a}^{\mu}(s), a=1,2,3$, which are orthogonal to $u^{\mu}$,

$$
e_{a}^{\mu} e_{b \mu}=-\delta_{a b}, \quad e_{a}^{\mu} u_{\mu}=0
$$

$Z^{\mu}$ are components of the Jacobi field in the coordinate basis, $\mathbf{Z}=Z^{\mu} \partial_{\mu}$. Since $Z^{\alpha} u_{\alpha}=0$, the field may be decomposed in the triad basis, $\mathbf{Z}=\sum_{a} Z_{a} \mathbf{e}_{a}$ and when the triad fields are kept fixed the functions $Z_{a}(s)$ are three scalar fields on $\gamma$. Equation (3) gets simplified when $e_{a}^{\mu}$ are parallelly transported along $\gamma,(D / d s) e_{a}^{\mu}=0$. The components $Z^{\mu}$ are expressed in terms of the scalars as

$$
Z^{\mu}=\sum_{a=1}^{3} Z_{a} e_{a}^{\mu}
$$

\footnotetext{
${ }^{2}$ We use here the traditional term 'twins' instead of the correct 'triplets'.
} 
Inserting (5) into (3) and multiplying the result by $e_{b}^{\mu}$ one arrives at the following linear equations for the three scalars $Z_{a}$,

$$
\frac{d^{2}}{d s^{2}} Z_{a}=-e_{a}^{\mu} R_{\mu \alpha \beta \gamma} u^{\alpha} u^{\beta} \sum_{b=1}^{3} e_{b}^{\gamma} Z_{b}
$$

At an initial point $p_{0}$ on $\gamma$ one gives initial values $e_{a}^{\mu}\left(p_{0}\right)$ satisfying (4), then the triad fields are uniquely determined by the parallel propagation. One seeks for a general solution to (6); for each $a$ it depends on two integration constants and one of them is a multiplicative factor. In the search for conjugate points one then imposes the initial condition $Z_{a}\left(p_{0}\right)=0$ and gets the desired Jacobi field. If one of the scalars, say $Z_{1}$, vanishes at a point $q$ on $\gamma, Z_{1}(q)=0$, one can identically set $Z_{2}=Z_{3} \equiv 0$ and the Jacobi field $Z^{\mu}(s)=Z_{1}(s) e_{1}^{\mu}(s)$ is zero at $q$; the points $p_{0}$ and $q$ are conjugate on $\gamma$.

In the next section we calculate and compare the lengths of a circular and a radial geodesic worldlines, determine Jacobi fields on them and their conjugate points in the case of the static spherically symmetric vacuum spacetime.

\section{Schwarzschild spacetime}

In this spacetime (in standard coordinates $t, r, \theta, \phi)$ geodesic circular orbits exist only for $r=r_{0}>3 M$, where $M$ is the energy of the spacetime. ${ }^{3}$ All the motions take place in the two-surface $\theta=\pi / 2$.

The twin A stays at rest at $r=r_{0}, \theta=\pi / 2$ and $\phi=\phi_{0}$. The twin B moves on a geodesic circular orbit $r=r_{0}$ with a constant linear velocity $v_{B}$ with respect to A. All the parameters of the B's geodesic are determined by $r_{0}$ (and $M$ ) and it is parameterized by its length,

$$
t-t_{0}=\left(\frac{r_{0}}{r_{0}-3 M}\right)^{1 / 2} s, \quad \phi-\phi_{0}=\frac{1}{r_{0}}\left(\frac{r_{0}}{r_{0}-3 M}\right)^{1 / 2} s
$$

and $\left(v_{B} / c\right)^{2}=M /\left(r_{0}-2 M\right) ; t_{0}$ is the moment when $\mathrm{A}, \mathrm{B}$ and $\mathrm{C}$ are together at the same place. The length of the geodesic B after making one full circle is determined from (7) as $\phi-\phi_{0}=2 \pi$ and is

$$
s_{B}=2 \pi r_{0}\left(\frac{r_{0}-3 M}{M}\right)^{1 / 2}
$$

and the corresponding coordinate time interval is

$$
\Delta t=2 \pi r_{0}\left(\frac{r_{0}}{M}\right)^{1 / 2}
$$

\footnotetext{
${ }^{3}$ Usually we put $c=G=1$; from time to time we shall explicitly display $c$ for clarity.
} 
By assumption at the point $P_{0}\left(t=t_{0}, r=r_{0}, \phi=\phi_{0}\right)$ the worldlines of the three twins intersect. The twin $\mathrm{C}$ moves on a radial geodesic emanating from $P_{0}$ with an initial radial velocity $(d r / d s)\left(t_{0}\right)=u>0$ directed outwards at $\theta=\pi / 2$ and $\phi=\phi_{0}$. At $t=t_{M}$ he reaches a maximum height $r=r_{M}$ where his radial velocity decreases to zero and he falls down along the ingoing radial geodesic until at $t=t_{1}$ he returns to $r=r_{0}$. Since the spacetime is static, the coordinate and proper time of the flight upwards are equal to these of falling down, i.e. $t_{1}-t_{M}=t_{M}-t_{0}$.

By assumption the three twins meet again at $P_{1}\left(t=t_{1}, r=r_{0}, \phi=\phi_{0}\right)$, i.e. $\mathrm{C}$ falls back to the initial place when B makes a full circle,

$$
t_{1}-t_{0}=2\left(t_{M}-t_{0}\right)=\Delta t
$$

The proper time $s_{A}$ of the static twin from $P_{0}$ to $P_{1}$ is

$$
s_{A}=\left(1-\frac{2 M}{r_{0}}\right)^{1 / 2} \Delta t=2 \pi r_{0}\left(\frac{r_{0}}{M}-2\right)^{1 / 2}
$$

and according to (1) is larger than $s_{B}[7,8]$, what shows that the circular geodesic B contains conjugate points between $P_{0}$ and $P_{1}$.

One then conjectures that the longest curve connecting $P_{0}$ and $P_{1}$ is the radial geodesic $\mathrm{C}$. To confirm the conjecture one computes the C's length and then shows that conjugate points, if exist on $\mathrm{C}$, are located beyond the segment $P_{0} P_{1}$. As is well known it is convenient to parameterize a radial geodesic in this spacetime by an angular parameter $\eta$,

$$
r(\eta)=r_{M} \cos ^{2} \eta=\frac{1}{2} r_{M}(\cos 2 \eta+1)
$$

where $-\alpha / 2 \leq \eta<\pi / 2$ and $r_{M}$ is the largest radial distance from the centre. By definition the initial point is $r(-\alpha / 2)=r_{0}$, then

$$
\cos \alpha=\frac{2 r_{0}}{r_{M}}-1 \quad \text { and } \quad 0<\alpha<\pi
$$

The return point is $r(\alpha / 2)=r_{0}$, the event horizon $r=2 M$ is at $\eta=\eta_{H}$ where $\cos ^{2} \eta_{H}=2 M / r_{M}$ and $r(\pi / 2)=0$. On the radial geodesic all geometrical quantities are determined by $r_{0}$ and the initial velocity $u$, or equivalently by the integral of energy. The twin $\mathrm{C}$ with mass $m$ and 4 -momentum $p^{\alpha}$ has conserved energy $E$ determined by the timelike Killing field, which is normalized to unity at the spatial infinity, $K^{\alpha}=\delta_{0}^{\alpha}$, hence $E / c=K^{\alpha} p_{\alpha}=p_{0}=\left(1-2 M /\left(c^{2} r\right)\right) m c d x^{0} / d s>0$.

We introduce dimensionless constant energy per unit rest energy, $k \equiv E /\left(m c^{2}\right)$, then

$$
\frac{d t}{d s}=k\left(1-\frac{2 M}{r}\right)^{-1}
$$


where $x^{0} \equiv t$. The geodesic equation for the radial coordinate is replaced by the universal integral of motion $u^{\alpha} u_{\alpha}=1$ which in the present case reads

$$
\left(\frac{d r}{d s}\right)^{2}=k^{2}+\frac{2 M}{r}-1
$$

At the starting point one has

$$
u^{2}=k^{2}+\frac{2 M}{r_{0}}-1
$$

and $r_{M}$ is determined from $k^{2}-1+\frac{2 M}{r_{M}}=0$, hence

$$
r_{M}=\frac{2 M}{1-k^{2}} \quad \text { and } \quad 0<k<1
$$

Since stable circular orbits require $r_{0}>3 M$ and $r_{M}>r_{0}$ one gets a lower limit $k^{2}>1 / 3$. The tangent vector $u^{\alpha}$ has, from (14) and (15), the nonvanishing components

$$
\frac{d t}{d s}=\frac{k \cos ^{2} \eta}{\cos ^{2} \eta-1+k^{2}}, \quad \frac{d r}{d s}=-\sqrt{1-k^{2}} \tan \eta
$$

the minus sign ensures that for $-\alpha / 2<\eta<0$ the velocity is directed outwards and for $\eta>0$ it is directed inwards. To obtain relations $t=t(\eta)$ and $s=s(\eta)$, one replaces (18) by derivatives with respect to $\eta$. The derivative $d s / d \eta$ is derived from the formula for $d s^{2}$ expressed as a function of $\eta$; one gets

$$
\left(\frac{d s}{d \eta}\right)^{2}=\frac{2 r_{M}^{3}}{M} \cos ^{4} \eta
$$

and employing that $d s / d \eta$ is always positive one finds

$$
s(\eta)=\left(\frac{r_{M}^{3}}{2 M}\right)^{1 / 2}\left[\frac{1}{2}(\sin 2 \eta+\sin \alpha)+\eta+\frac{\alpha}{2}\right] .
$$

This immediately yields the length of $\mathrm{C}$ from $P_{0}$ to $P_{1}$,

$$
s_{C}=s\left(\frac{\alpha}{2}\right)=\left(\frac{r_{M}^{3}}{2 M}\right)^{1 / 2}\left[\frac{2 r_{0}}{r_{M}} \sqrt{\frac{r_{M}}{r_{0}}-1}+\arccos \left(\frac{2 r_{0}}{r_{M}}-1\right)\right]
$$


As concerns $t(\eta)$ one has $d t / d \eta=(d t / d s)(d s / d \eta)$ and substituting from (18) and (19) one gets

$$
\begin{aligned}
t(\eta)-t_{0}= & \int_{-\frac{\alpha}{2}}^{\eta} k\left(\frac{r_{M}^{3}}{2 M}\right)^{1 / 2} \frac{(\cos 2 \eta+1)^{2}}{\cos 2 \eta+2 k^{2}-1} d \eta \\
= & k\left(\frac{r_{M}^{3}}{2 M}\right)^{1 / 2}\left[\left(3-2 k^{2}\right)\left(\eta+\frac{\alpha}{2}\right)+\frac{1}{2}(\sin 2 \eta+\sin \alpha)\right] \\
& +2 M \ln \left(\frac{b+\tan \eta}{b-\tan \eta}\right)-2 M \ln \left(\frac{b-\tan \alpha / 2}{b+\tan \alpha / 2}\right),
\end{aligned}
$$

where $^{4} b=k\left(1-k^{2}\right)^{-1 / 2}$. Notice that at the event horizon $\sin \eta_{H}=+k$, then $\tan \eta_{H}=b$ and $t \rightarrow+\infty$ as it should be. Accordingly, the coordinate time interval of the flight back and forth is

$$
t(\alpha / 2)-t_{0}=k\left(\frac{r_{M}^{3}}{2 M}\right)^{1 / 2}\left[\left(3-2 k^{2}\right) \alpha+\sin \alpha\right]+4 M \ln \left(\frac{b+\left(\frac{r_{M}}{r_{0}}-1\right)^{1 / 2}}{b-\left(\frac{r_{M}}{r_{0}}-1\right)^{1 / 2}}\right) .
$$

Here

$$
b^{2}-\left(\frac{r_{M}}{r_{0}}-1\right)=\frac{1}{1-k^{2}}\left(1-\frac{2 M}{r_{0}}\right)>0,
$$

hence the denominator of the fraction under the logarithm is always positive. The interval (23) must be equal to $\Delta t$ given in (9) and this gives a transcendental algebraic equation for the energy $k$ or equivalently the initial radial velocity $u$ in terms of the parameter $r_{0}$. Introducing for convenience a parameter $R=r_{0} /(2 M)>3 / 2$ and replacing the unknown $k\left(r_{0}\right)$ by $x=r_{0} / r_{M}=R\left(1-k^{2}\right)=1-u^{2} R$ one finally gets an equation for $x\left(r_{0}\right)$ (or $\left.x(R)\right)$,

$$
\begin{aligned}
2 \sqrt{2} \pi R^{3 / 2}= & \left(1-\frac{x}{R}\right)^{1 / 2}\left(\frac{R}{x}\right)^{3 / 2}\left[\left(1+\frac{2 x}{R}\right) \alpha+2 \sqrt{x-x^{2}}\right] \\
& +2 \ln \left(\frac{\sqrt{R-x}+\sqrt{1-x}}{\sqrt{R-x}-\sqrt{1-x}}\right),
\end{aligned}
$$

where, according to (13), $\cos \alpha=2 x-1$. It may be shown that for each value of $R>3 / 2$ there is only one solution of the equation in the interval $0<x<1$. Inserting

\footnotetext{
4 Clearly formulae (20) and (22) have already been known in various versions, we rederive them here for the reader's convenience.
} 
Table 1 The ratio $s_{C} / s_{A}$ for 5 values of $x(R)$

\begin{tabular}{lll}
\hline$R$ & $x$ & $s_{C} / s_{A}$ \\
\hline 1,5 & 0,545246 & 1,19878 \\
1,6 & 0,534476 & 1,17554 \\
2 & 0,508559 & 1,12061 \\
4 & 0,472995 & 1,04849 \\
100 & 0,44932 & 1,00162 \\
\hline
\end{tabular}

this value of $x(R)$ into (21) one gets the ratio of the twins' proper times,

$$
\frac{s_{C}}{s_{A}}=\frac{1}{\sqrt{2} \pi}\left(1-\frac{1}{R}\right)^{-1 / 2} x^{-3 / 2}\left[\sqrt{x-x^{2}}+\frac{1}{2} \arccos (2 x-1)\right] .
$$

Equation (24) was numerically solved for five values of $R$ consecutively growing by 0,1 from 1,5 to 2 and for all integers from $R=2$ to 100 . The value of $x(R)$ is a monotonic slowly diminishing function of $R$ (we did not attempt to fit it by an analytic expression) and decreases from 0,545246 for $R=1,5$ to 0,44932 for $R=100$. It is hard to analytically establish from Eq. (25) which proper time is larger and we compute their numerical values. In Table 1 we give the ratio $s_{C} / s_{A}$ for some chosen values of $x(R)$. As it should be expected, the farther the point $r_{0}$ is from the event horizon the smaller the ratio $s_{C} / s_{A}$ is and always the radial geodesic $\mathrm{C}$ is longer than the non-geodesic curve A. That $s_{C}>s_{A}$ was previously found in a special case in [12].

Both the geodesic curves B and C satisfy the conditions of Proposition 4, hence there do exist conjugate points and to identify them we now find Jacobi fields on these worldlines.

\subsection{The timelike radial geodesic $\mathrm{C}$}

An alternative expression to formula (18) for the tangent vector to $\mathrm{C}$ in terms of the coordinate $r$ is

$$
u^{\alpha}=\left[\frac{k r}{r-2 M}, \varepsilon\left(\frac{2 M}{r}-1+k^{2}\right)^{1 / 2}, 0,0\right]
$$

where $\varepsilon=+1$ on the outwards directed segment of $\mathrm{C}$ and $\varepsilon=-1$ on the inwards directed piece. The parallelly propagated and orthogonal to $\mathrm{C}$ orthonormal spacelike triad is chosen as

$$
\begin{aligned}
e_{1}^{\mu} & =\left[\frac{\varepsilon r}{r-2 M}\left(\frac{2 M}{r}-1+k^{2}\right)^{1 / 2}, k, 0,0\right] \\
& =\left[-\left(1-k^{2}\right)^{1 / 2} \frac{\sin \eta \cos \eta}{\cos ^{2} \eta-1+k^{2}}, k, 0,0\right], \\
e_{2}^{\mu} & =\left[0,0, \frac{1}{r}, 0\right], \quad e_{3}^{\mu}=\left[0,0,0, \frac{1}{r}\right]
\end{aligned}
$$


with $\varepsilon$ as in (26). The geodesic deviation equation for the scalars $Z_{a}$ reduces in the present case to

$$
\begin{aligned}
\frac{d^{2}}{d s^{2}} Z_{1} & =\frac{2 M}{r^{3}} Z_{1}, \\
\frac{d^{2}}{d s^{2}} Z_{2} & =-\frac{M}{r^{3}} Z_{2}, \\
\frac{d^{2}}{d s^{2}} Z_{3} & =-\frac{M}{r^{3}} Z_{3} .
\end{aligned}
$$

Simplicity of these equations is deceptive since their left hand sides contain derivatives with respect to the arc length instead of $r$ or $\eta$. One replaces these second derivatives by derivatives with respect to $\eta$,

$$
\begin{aligned}
\frac{d Z_{a}}{d s} & =\frac{p}{\cos 2 \eta+1} \frac{d Z_{a}}{d \eta}, \\
\frac{d^{2} Z_{a}}{d s^{2}} & =\frac{p^{2}}{(\cos 2 \eta+1)^{2}}\left[\frac{d^{2} Z_{a}}{d \eta^{2}}+\frac{2 \sin 2 \eta}{\cos 2 \eta+1} \frac{d Z_{a}}{d \eta}\right],
\end{aligned}
$$

where $p \equiv\left(r_{M}^{3} / 2 M\right)^{-1 / 2}$. Then the Jacobi equations read

$$
\begin{aligned}
& \frac{d^{2} Z_{1}}{d \eta^{2}}+\frac{2 \sin 2 \eta}{\cos 2 \eta+1} \frac{d Z_{1}}{d \eta}-\frac{8}{\cos 2 \eta+1} Z_{1}=0 \\
& \frac{d^{2} Z_{2}}{d \eta^{2}}+\frac{2 \sin 2 \eta}{\cos 2 \eta+1} \frac{d Z_{2}}{d \eta}+\frac{4}{\cos 2 \eta+1} Z_{2}=0
\end{aligned}
$$

and for $Z_{3}$ one has an equation identical to that for $Z_{2}$. To solve Eq. (34) one introduces a change of the independent variable, $x=\cos 2 \eta$ (not to be confused with the parameter $r_{0} / r_{M}$ introduced earlier), then $u=(x+1) / 2$ and substitutes $Z_{1}(u)$ $=4 u^{2} G(u)$. Then (34) reduces to a special case of Gauss hypergeometric equation which is solved in elementary functions. After some manipulations one gets a generic solution for $Z_{1}$,

$$
Z_{1}(\eta)=C_{1} \tan \eta+C_{2}\left(3 \eta \tan \eta-\frac{3}{2} \pi \tan \eta-\cos ^{2} \eta+3\right)
$$

In the case of Eq. (35) one performs the same operations as previously and finally substitutes $G(u)=\frac{1}{u} Y(u)$, this yields an equation

$$
u(u-1) \frac{d^{2} Y}{d u^{2}}+\left(2 u-\frac{3}{2}\right) \frac{d Y}{d u}=0
$$

which is also solved in elementary functions. The general solution is

$$
Z_{2}(\eta)=C^{\prime} \sin 2 \eta+C^{\prime \prime}(\cos 2 \eta+1)
$$


and an analogous formula holds for $Z_{3}$. A general Jacobi field on a radial geodesic was earlier found by Fuchs [13]. He used a different basis triad whose explicit form in terms of $r$ or $\eta$ was not given, hence it is not easy to compare his solutions to ours.

We now seek for conjugate points on $\mathrm{C}$. The special Jacobi scalars which vanish at $P_{0}(\eta=-\alpha / 2)$ are then

$$
\begin{aligned}
& Z_{1}(\eta)=C_{1}\left[\left(\frac{3}{2} \alpha+\frac{4 \cos \alpha-\cos ^{2} \alpha+5}{2 \sin \alpha}+3 \eta\right) \tan \eta-\cos ^{2} \eta+3\right] \\
& Z_{2}(\eta)=C_{2}\left[\sin 2 \eta+\frac{\sin \alpha}{\cos \alpha+1}(\cos 2 \eta+1)\right]
\end{aligned}
$$

and $Z_{3}$ is given by the same formula as that for $Z_{2}$ with $C_{2}$ replaced by $C_{3} ; C_{1}, C_{2}$ and $C_{3}$ are arbitrary constants. Functions $Z_{2}$ and $Z_{3}$ vanish at $\eta=\pi / 2$, i.e. at $r=0$. In this sense the curvature singularity is a point conjugate on the radial geodesic to any point $P_{0}$. For $-\alpha / 2 \leq \eta<\pi / 2$ the equation $Z_{2}(\eta)=0$ is reduced to

$$
\tan \eta=-\frac{\sin \alpha}{\cos \alpha+1}=-\sqrt{\frac{r_{M}}{r_{0}}-1}<0
$$

and its solution belongs to the interval $-\pi / 2<\eta<0$ where tangens is monotonic, hence the only solution is $\eta=-\alpha / 2$. In sum, the Jacobi field $Z^{\mu}=Z_{2} e_{2}^{\mu}+Z_{3} e_{3}^{\mu}$ is different from zero on the radial geodesic segment from any $P_{0}$ to the event horizon.

As concerns $Z_{1}(\eta)$ one easily shows that the ratio $Z_{1} / C_{1} \geq 2$ in the interval $0 \leq \eta<\pi / 2$ and possible zeros may be only on the outgoing segment of the geodesic, $-\alpha / 2<\eta<0$. One views $Z_{1}$ as a function of two variables, $Z_{1}(\eta, \alpha)$ with $\alpha \in(0, \pi)$. For any fixed value of $\eta$ one has $\alpha \in(-2 \eta, \pi)$ and in this interval $Z_{1}(\eta, \alpha)$ grows since

$$
\frac{\partial Z_{1}}{\partial \alpha}=\cot ^{2} \alpha / 2 \cos ^{2} \alpha / 2 \tan (-\eta)>0 .
$$

From $Z_{1}(-\alpha / 2, \alpha)=0$ one infers that $Z_{1} / C_{1}$ is positive for $-\alpha / 2<\eta<0$, thence the function has no zeros for $-\alpha / 2<\eta<\pi / 2$.

This completes the proof that a timelike radial geodesic consisting of an outgoing segment and an ingoing one contains no conjugate points and is the longest curve joining points $P_{0}$ and $P_{1}$ outside the event horizon. Clearly the same statement concerning non-existence of conjugate points applies to any timelike radial geodesic which is purely outgoing or ingoing.

\subsection{The timelike circular geodesic B}

It is rather surprising that solving the equations for Jacobi fields is in this case much harder than for a radial geodesic. One immediately gets the vector $u^{\alpha}$ tangent to the geodesic B from its parametric form (7). A triad of spacelike orthonormal vector vector fields on B satisfying (4) and parallelly transported along it may be chosen as 


$$
\begin{aligned}
e_{1}^{\mu}= & {\left[-\left(\frac{M r_{0}}{\left(r_{0}-2 M\right)\left(r_{0}-3 M\right)}\right)^{1 / 2} \sin q s,\left(\frac{r_{0}-2 M}{r_{0}}\right)^{1 / 2} \cos q s, 0,\right.} \\
& \left.\frac{-1}{r_{0}}\left(\frac{r_{0}-2 M}{r_{0}-3 M}\right)^{1 / 2} \sin q s\right], \\
e_{2}^{\mu}= & {\left[0,0, \frac{1}{r_{0}}, 0\right], } \\
e_{3}^{\mu}= & {\left[\left(\frac{M r_{0}}{\left(r_{0}-2 M\right)\left(r_{0}-3 M\right)}\right)^{1 / 2} \cos q s,\left(\frac{r_{0}-2 M}{r_{0}}\right)^{1 / 2} \sin q s, 0,\right.} \\
& \left.\frac{1}{r_{0}}\left(\frac{r_{0}-2 M}{r_{0}-3 M}\right)^{1 / 2} \cos q s\right],
\end{aligned}
$$

where $q^{2}=M / r_{0}^{3}$. Employing

$$
u^{\alpha}=\left(r_{0}-3 M\right)^{-1 / 2}\left(r_{0}^{1 / 2} \delta_{0}^{\alpha}+\frac{M^{1 / 2}}{r_{0}} \delta_{3}^{\alpha}\right)
$$

and denoting

$$
\beta=3 \frac{r_{0}-2 M}{r_{0}-3 M}
$$

one arrives at the following form of Eq. (6) for the scalars $Z_{a}(s)$,

$$
\begin{aligned}
\frac{d^{2}}{d s^{2}} Z_{1} & =q^{2}\left[\left(\beta \cos ^{2} q s-1\right) Z_{1}+\beta Z_{3} \sin q s \cos q s\right], \\
\frac{d^{2}}{d s^{2}} Z_{2} & =-\frac{M}{r_{0}^{2}\left(r_{0}-3 M\right)} Z_{2}, \\
\frac{d^{2}}{d s^{2}} Z_{3} & =q^{2}\left[\beta Z_{1} \sin q s \cos q s+\left(\beta \sin ^{2} q s-1\right) Z_{3}\right] .
\end{aligned}
$$

It will be shown that Jacobi fields spanned on the basis vectors $e_{1}^{\mu}$ and $e_{3}^{\mu}$ do not give rise to a point conjugate to $P_{0}$ on the segment $P_{0} P_{1}$ and in this sense they are irrelevant to the twin paradox. We therefore postpone solving Eqs. (45) and (47) to the "Appendix" and in the current subsection we discuss consequences of the equation for $Z_{2}$.

The general solution for Eq. (46) reads

$$
Z_{2}=C^{\prime} \sin p_{0} s+C^{\prime \prime} \cos p_{0} s
$$

where

$$
p_{0}^{2}=\frac{M}{r_{0}^{2}\left(r_{0}-3 M\right)}
$$


and $C^{\prime}$ and $C^{\prime \prime}$ are integration constants. The special Jacobi field proportional to $e_{2}^{\mu}$ and vanishing at $P_{0}(s=0)$ is simply $Z^{\mu}=C \delta_{2}^{\mu} \sin p_{0} s$. The field generates infinite number of points conjugate to $P_{0}$ which are located at

$$
s_{n}=n \pi\left(\frac{r_{0}-3 M}{M}\right)^{1 / 2} r_{0},
$$

$n=1,2, \ldots$. The nearest to $P_{0}$ conjugate point $Q$ is at $s_{Q}=s_{B} / 2$, that is half way between $P_{0}$ and the endpoint $P_{1}$. Notice that $P_{1}$ is also conjugate to both $P_{0}$ and to $Q$.

One conjectures that analogous features occur in Reissner-Nordström spacetime, this will be shown in a forthcoming paper.

\section{Summary}

In this work we give explicit forms of generic Jacobi fields and conjugate points generated by these fields on physically distinguished timelike geodesic curves in Schwarzschild spacetime. These are circular orbits and radial curves going back and forth. Applying the theorem that the longest timelike curve joining two given points is a geodesic free of points conjugate to its endpoints and lying between the ends, we show that in this spacetime the longest worldline is any radial geodesic. This outcome may be physically interpreted in terms of the famous twin paradox in the spacetime. Considering a number of twins following different worldlines with common endpoints one finds that contrary to a recent conjecture the relative ageing of the twins is determined neither by their velocities (with respect to a static observer) nor their accelerations (in the case of non-geodesic motions). The twin travelling on a radial geodesic in Schwarzschild spacetime is at the reunion the oldest one among all the twins. Yet the twin moving on a geodesic circular orbit is younger than the static twin.

Acknowledgments I am deeply indebted to Zdzisław Golda for solving a differential equation with the aid of Mathematica and to Sebastian Szybka for making some analytic and numerical computations. This work was supported by a grant from the John Templeton Foundation.

Open Access This article is distributed under the terms of the Creative Commons Attribution License which permits any use, distribution, and reproduction in any medium, provided the original author(s) and the source are credited.

\section{Appendix}

Here we solve Eqs. (45) and (47) and get a generic Jacobi field on the timelike circular geodesic $\mathrm{B}$ what allows one for the search for all conjugate points on this geodesic. To solve for $Z_{1}$ and $Z_{3}$ one first introduces a dimensionless independent variable $x=q s$ [ $q$ is defined after Eq. (43)] and then replaces the two coupled equations (45) and (47) by one equation for $Z_{1}$ by eliminating $Z_{3}$. As a result one gets a fourth order equation 


$$
\begin{gathered}
Z_{1}^{(I V)}-4 \cot 2 x Z_{1}^{\prime \prime \prime}+\left(\frac{8}{\sin ^{2} 2 x}-\beta-2\right) Z_{1}^{\prime \prime}+\frac{2}{\sin 2 x}[\beta+(\beta-2) \cos 2 x] Z_{1}^{\prime} \\
-\left[\frac{4}{\sin ^{2} 2 x}(\beta \cos 2 x+\beta-2)+3-\beta\right] Z_{1}=0
\end{gathered}
$$

with $Z_{1}^{\prime}=d Z_{1} / d x$ etc. The equation has four linearly independent special solutions ${ }^{5}$ $Z_{1 N}, N=1,2,3,4$ and these read

$$
\begin{aligned}
& Z_{11}=\sin x, \\
& Z_{12}=2 \cos x+\beta x \sin x, \\
& Z_{13}= \begin{cases}2 \sin x \sin (\sqrt{4-\beta} x)+\sqrt{4-\beta} \cos x \cos (\sqrt{4-\beta} x), & 3<\beta<4, \\
x \cos x+x^{2} \sin x, & \beta=4, \\
2 \sin x \sinh (\sqrt{\beta-4} x)+\sqrt{\beta-4} \cos x \cosh (\sqrt{\beta-4} x), & \beta>4,\end{cases} \\
& Z_{14}= \begin{cases}2 \sin x \cos (\sqrt{4-\beta} x)-\sqrt{4-\beta} \cos x \sin (\sqrt{4-\beta} x), & 3<\beta<4, \\
4 x^{3} \sin x+\left(3+6 x^{2}\right) \cos x, & \beta=4, \\
2 \sin x \cosh (\sqrt{\beta-4} x)+\sqrt{\beta-4} \cos x \sinh (\sqrt{\beta-4} x), \beta>4,\end{cases}
\end{aligned}
$$

$x=q s$. One sees that $\beta=4$, corresponding to $r_{0}=6 M$, the innermost stable circular orbit (ISCO), is distinguished. The general solution to (A.1) is then

$$
Z_{1}=\sum_{N=1}^{4} C_{N} Z_{1 N}
$$

with arbitrary constants $C_{N}$. The scalar $Z_{3}$ is determined from Eq. (45) as action of a linear operator on $Z_{1}$,

$$
\begin{aligned}
Z_{3} & =\left(\frac{2}{\beta \sin 2 x} \frac{d^{2}}{d x^{2}}-\cot 2 x-\frac{\beta-2}{\beta \sin 2 x}\right) Z_{1} \equiv L Z_{1}, \\
\text { or } \quad Z_{3} & =\sum_{N=1} C_{N} L Z_{1 N} \equiv \sum_{N=1} C_{N} Z_{3 N} .
\end{aligned}
$$

\footnotetext{
5 The four solutions were found by Z. Golda by manipulating with the equation with the aid of Mathematica. There is a first integral generated by the timelike Killing vector field (we discuss such integrals in Part II) but it turns out to be of little use.
} 
Explicitly, the special solutions $Z_{3 N}$ are

$$
\begin{aligned}
& Z_{31}=-\cos x, \\
& Z_{32}=2 \sin x-\beta x \cos x, \\
& Z_{33}= \begin{cases}-2 \cos x \sin (\sqrt{4-\beta} x)+\sqrt{4-\beta} \sin x \cos (\sqrt{4-\beta} x), & 3<\beta<4, \\
x \sin x-x^{2} \cos x, & \beta=4, \\
\sqrt{\beta-4} \sin x \cosh (\sqrt{\beta-4} x)-2 \cos x \sinh (\sqrt{\beta-4} x), & \beta>4,\end{cases} \\
& Z_{34}= \begin{cases}-2 \cos x \cos (\sqrt{4-\beta} x)-\sqrt{4-\beta} \sin x \sin (\sqrt{4-\beta} x), & 3<\beta<4, \\
3\left(1+2 x^{2}\right) \sin x-4 x^{3} \cos x, & \beta=4, \\
\sqrt{\beta-4} \sin x \sinh (\sqrt{\beta-4} x)-2 \cos x \cosh (\sqrt{\beta-4} x), & \beta>4 .\end{cases}
\end{aligned}
$$

Altogether the general Jacobi field on B depends on 6 arbitrary constants and takes the form

$$
Z^{\mu}(s)=\sum_{N=1}^{4} C_{N}\left(Z_{1 N} e_{1}^{\mu}+Z_{3 N} e_{3}^{\mu}\right)+\left(C^{\prime} \sin p_{0} s+C^{\prime \prime} \cos p_{0} s\right) e_{2}^{\mu}
$$

We have seen in Sect. 3.2 that the special Jacobi field proportional to $e_{2}^{\mu}$ generates an infinite sequence of points conjugate to $P_{0}$ of which the first two are located on the segment $P_{0} P_{1}$, hence the field does account for the fact that the circular geodesic $\mathrm{B}$ is shorter between $P_{0}$ and $P_{1}$ than the radial geodesic $\mathrm{C}$ and the non-geodesic curve A. We now establish whether a Jacobi field spanned on the basis vectors $e_{1}^{\mu}$ and $e_{3}^{\mu}$ may also give rise to a conjugate point. The procedure is simple and laborious. One must study separate cases $3<\beta<4, \beta=4$ and $\beta>4$. In the special case $\beta=4$ i.e. the ISCO geodesic line, the initial condition $Z_{1}(0)=0=Z_{3}(0)$, yields $C_{2}=3 C_{1} / 4$ and $C_{4}=-C_{1} / 2$ with arbitrary $C_{1}$ and $C_{3}$. It turns out that the scalar fields $Z_{1}(q s)$ and $Z_{3}(q s)$ have no common roots for $s>0$. In the generic case, $\beta \neq 4$, one again gets that the functions $Z_{1}$ and $Z_{3}$ vanishing at $s=0$ depend on arbitrary $C_{1}$ and $C_{3}$, now with

$$
C_{2}=-\frac{1}{2}|\beta-4|^{1 / 2} C_{3}, \quad C_{4}=-\frac{1}{2} C_{1}
$$

For $\beta>4$, i.e. for $3 M<r_{0}<6 M$, one again finds that $Z_{1}$ and $Z_{3}$ do not possess common roots for $s>0$. Finally, for $r_{0}>6 M$ one finds that the scalars

$$
Z_{1}=2 Z_{11}-Z_{14} \quad \text { and } \quad Z_{3}=2 Z_{31}-Z_{34}
$$

vanish at

$$
s_{n}=\frac{2 n \pi}{q \sqrt{4-\beta}}=2 n \pi\left(\frac{r_{0}^{3}}{M}\right)^{1 / 2}\left(\frac{r_{0}-3 M}{r_{0}-6 M}\right)^{1 / 2}, \quad n=1,2, \ldots
$$


Accordingly, besides the sequence (50) found previously, there is a second infinite sequence of points $Q_{n}^{\prime}\left(s_{n}\right)$ conjugate to $P_{0}$ on the geodesic B. To check whether the first of these, $Q_{1}^{\prime}\left(s_{1}\right)$, is located on the segment $P_{0} P_{1}$ we compare the value of $s_{1}$ with the length $s_{B}$ of the curve from $P_{0}$ to $P_{1}$. From (8) one gets

$$
\frac{s_{1}}{s_{B}}=\left(\frac{r_{0}}{r_{0}-6 M}\right)^{1 / 2}>1
$$

showing that all the points $Q_{n}^{\prime}$ lie beyond the relevant segment. One concludes that these conjugate points do not account for the fact that the circular geodesic $\mathrm{B}$ has a non-maximal length.

\section{References}

1. Lichtenegger, H.I.M., Iorio, L.: The twin paradox and Mach's principle [arXiv:0910.1929v1]

2. Iorio, L.: An analytical treatment of the clock paradox in the framework of the special and general theories of relativity. Found. Phys. Lett. 18, 1 (2005) [arXiv:physics/0405038]

3. Iorio, L.: On the clock paradox in the case of circular motion of the moving clock. Eur. J. Phys. 26, 535 (2005) [arXiv:physics/0406139]

4. Jones, P., Wanex, L.F.: The clock paradox in a static homogeneous gravitational field. Found. Phys. Lett. 19, 75 (2006) [arXiv:physics/0604025]

5. Dolby, C.E., Gull, S.F.: On radar time and the twin paradox, Am. J. Phys. 69, 1257 (2001) [arXiv:grqc/0104077v2]

6. Székely, G.: A geometrical characterization of the twin paradox and its variants. Studia Logica 95, 161 (2010) [arXiv:0807.1813v2]

7. Abramowicz M.A., Bajtlik S., Kluźniak W. (2007) The twin paradox on the photon sphere. Phys. Rev. A 75, 044101

8. Abramowicz, M.A., Bajtlik, S.: Adding to the paradox: the accelerated twin is older [arXiv: 0905.2428v1]

9. Stephani, H.: Relativity, An Introduction to Special and General Relativity, 3rd edn, par. 3.4. Cambridge University Press, Cambridge (2004)

10. Hawking, S.W., Ellis, G.F.R.: The Large Scale Structure of Space-Time. Cambridge University Press, Cambridge (1973)

11. Wald, R.M.: General Relativity. University of Chicago Press, Chicago (1984)

12. Grøn, Ø., Braeck, S.: The twin paradox in a cosmological context. Eur. Phys. J. Plus 126, 79 (2011) [arXiv:0909.5364v4]

13. Fuchs, H.: Solutions of the equations of geodesic deviation for static spherical symmetric spacetimes. Ann. d. Physik 40, 231 (1983) 